\title{
SINGULAR VALUE AND NORM \\ INEQUALITIES OF DAVIDSON-POWER TYPE
}

\section{WASIM AUDEH}

Abstract. Let $A, B, X$ and $Y$ be $n \times n$ complex matrices such that $A$ and $B$ are positive semidefinite, then

$$
\|A X+Y B\| \leqslant \frac{1}{4}\left(\left\|W_{1}\right\|+\left\|W_{2}\right\|+W_{4}\right),
$$

where

$$
\begin{gathered}
W_{1}=A+A^{1 / 2}\left|X^{*}\right|^{2} A^{1 / 2}, \\
W_{2}=B+B^{1 / 2}|Y|^{2} B^{1 / 2}, \\
W_{3}=A^{1 / 2} X B^{1 / 2}+A^{1 / 2} Y B^{1 / 2}
\end{gathered}
$$

and

$$
W_{4}=\sqrt{\left(\left\|W_{1}\right\|-\left\|W_{2}\right\|\right)^{2}+4\left\|W_{3}\right\|^{2}} .
$$

Multiple results are given in this paper.

Mathematics subject classification (2020): 15A18, 15A42, 15A60, 47A30, 47B15.

Keywords and phrases: Concave function, positive semidefinite matrix, singular value, unitarily invariant norm, inequality.

\section{REFERENCES}

[1] W. AUDEH, Some generalizations for singular value inequalities of compact operators, Adv. Oper. Theory 6 (2021).

[2] W. AUDEH, Singular value inequalities and applications, Positivity 25 (2020), 843-852.

[3] W. AUDEH, Generalizations for singular value and arithmetic-geometric mean inequalities of operators, J. Math. Anal. Appl. 489 (2020), 1-8.

[4] W. AUDEH, Generalizations for singular value inequalities of operators, Adv. Oper. Theory 5 (2020), 371-381.

[5] W. Audeh, F. Kittaneh, Singular value inequalities for compact operators, Linear Algebra Appl. 437 (2012), 2516-2522.

[6] R. Bhatia, Matrix Analysis, Springer, New York, 1997.

[7] R. BhatiA AND F. KitTANEH, On the singular values of a product of operators, SIAM J. Matrix Anal. Appl. 11 (1990), 272-277.

[8] J. C. Bourin, A matrix subadditivity inequality for symmetric norms, Proc. Amer. Math. Soc. 138 (2009), 495-504.

[9] K. DAVIDSON, S. C. Power, Best approximation in $C^{*}$-algebras, J. Reine Angew. Math. 368 (1986), 43-62.

[10] O. HirzallaH, Inequalities for sums and products of operators, Linear Algebra Appl. 407 (2005), $32-42$.

[11] R. A. Horn, And C. R. Johnson, Matrix Analysis, 2nd edition, Cambridge University Press, 2013.

[12] J. C. Hou, H. K. DU, Norm inequalities of positive operator matrices, Integral equations operator theory 22 (1995), 281-294.

[13] F. Kittaneh, Norm inequalities for certain operator sums, J. Funct. Anal. 143 (1997), 337-348.

[14] F. KitTANEH, Norm inequalities for sums of positive operators, J. Operator theory 48 (2002), 95-103. 
[15] X. ZHAN, Singular values of differences of positive semidefinite matrices, SIAM J. Matrix Anal. Appl. 22 (2002), 819-823. 